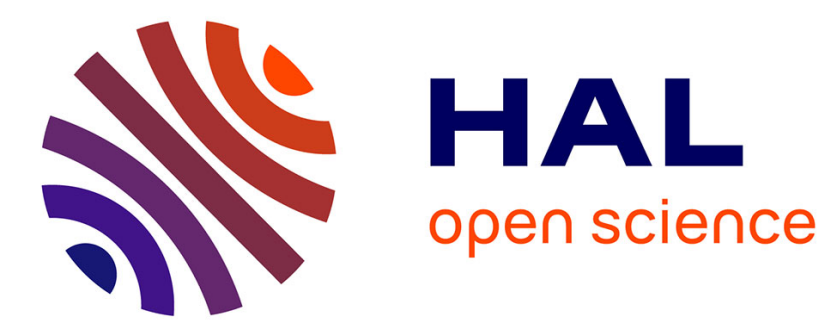

\title{
Dynamical splitting of cubic crystal field levels in rare-earth cage compounds
}

\author{
Mehdi Amara
}

\section{To cite this version:}

Mehdi Amara. Dynamical splitting of cubic crystal field levels in rare-earth cage compounds. Physical Review B, 2019, 99 (17), pp.174405. 10.1103/PhysRevB.99.174405 . hal-02125893

\section{HAL Id: hal-02125893 \\ https://hal.science/hal-02125893}

Submitted on 10 May 2019

HAL is a multi-disciplinary open access archive for the deposit and dissemination of scientific research documents, whether they are published or not. The documents may come from teaching and research institutions in France or abroad, or from public or private research centers.
L'archive ouverte pluridisciplinaire HAL, est destinée au dépôt et à la diffusion de documents scientifiques de niveau recherche, publiés ou non, émanant des établissements d'enseignement et de recherche français ou étrangers, des laboratoires publics ou privés. 


\title{
Dynamical splitting of cubic crystal field levels in rare-earth cage compounds
}

\author{
Mehdi Amara* \\ Univ. Grenoble Alpes, CNRS, Grenoble INP, Institut Néel, 38000 Grenoble, France
}

(Received 21 December 2018; revised manuscript received 14 March 2019; published 7 May 2019)

\begin{abstract}
The crystalline electric field (CEF) influence is usually described by considering an ideally symmetrical rare-earth site. In the case of cage compounds, ample excursions of the rare earth inside the cage require an adapted CEF description. A corrective, position-dependent, CEF term accounts for the deviation from the perfect symmetry. In the paramagnetic range, a CEF level with orbital degeneracy thus acquires a width reflecting the rare-earth spatial distribution. In the case, frequent in cubic systems, of an orbitally degenerate CEF ground state at the center, this width introduces an additional energy scale, influential at low temperature. A spherical simplification allows to identify the major consequences of a cage-split ground multiplet: a Schottky-type anomaly appears in the specific heat with associated reduction of the magnetic entropy and alteration of the magnetic properties. Concomitantly, a centrifugal Jahn-Teller effect develops that expands the distribution of the magnetic ion and softens the rattling phonons. These effects are confronted with anomalous paramagnetic properties of rare-earth cage compounds, notably rare-earth-filled skutterudites and hexaborides.
\end{abstract}

DOI: 10.1103/PhysRevB.99.174405

\section{INTRODUCTION}

Impulsed by an interest in thermoelectric applications, the investigation of metallic cage compounds has soared during the last decade. In these systems, atoms are enclosed in oversized cages, allowing relatively large excursions from their average positions. This "rattling" of the guest is supposed to reduce the thermal conductivity, thus improving the thermoelectric efficiency of these metals. In some crystallographic structures, the cage can accommodate a rare-earth ion, giving rise to specific magnetic properties. The most investigated rare-earth cage compounds are filled skutterudites, that crystallize according to the $\mathrm{LaFe}_{4} \mathrm{P}_{12}$-type structure [1]. These compounds display a variety of intriguing features, as the heavy fermion and superconductor $\mathrm{PrOs}_{4} \mathrm{Sb}_{12}$ [2], or the metal-insulator transition (MI) in $\mathrm{PrRu}_{4} \mathrm{P}_{12}$ [3], the nonmagnetic ordering of $\mathrm{PrFe}_{4} \mathrm{P}_{12}$ [4], etc. These unconventional behaviors echo those of an extensively investigated, but still elusive, series of rare-earth cage compounds: the rare-earth hexaborides. Among them, the most enigmatic $\mathrm{CeB}_{6}$ features a nonconventional ordering $[5,6]$.

To understand the properties of rare-earth compounds, accounting for the effect of the crystalline electric field (CEF) is essential. The CEF reflects the anisotropic environment of the $4 f$ ion and lifts, at least partly, the orbital degeneracy of the $4 f$ shell. As a result, the $2 J+1$ degenerate ground-state multiplet is decomposed to form the CEF scheme, according to the point symmetries of the rare-earth site: this is a notorious and early success in the application of group theory to quantum mechanics [7]. In the temperature range where magnetic phenomena occur, this CEF scheme is considered as a stable feature, used as a starting point for any microscopic description of the paramagnetic or ordering properties. In

*mehdi.amara@neel.cnrs.fr rare-earth intermetallic compounds, the most effective means, for the experimental determination of the CEF scheme, is neutron spectroscopy. However, in many instances of rare-earth cage compounds, difficulties emerge at the stage of the neutron spectroscopy investigation, notably for rare-earth-filled skutterudites. For instance, the inelastic spectra of $\operatorname{PrRu}_{4} \mathrm{P}_{12}$ are inconsistent with the $T_{h}$ symmetry of the Pr site below the MI transition, displaying more CEF transitions than allowed, and show a spectacular broadening of these excitations above [3]. In $\mathrm{PrFe}_{4} \mathrm{P}_{12}$, well-defined CEF excitations appear only in the ordered state [8], while in $\operatorname{PrOs}_{4} \mathrm{P}_{12}$, they vanish very rapidly with increasing the temperature [9]. For some light rare-earth hexaborides, neutron diffraction and Raman scattering investigations where successfully used for determining the CEF scheme [10,11]. In $\mathrm{CeB}_{6}, \mathrm{PrB}_{6}$, and $\mathrm{NdB}_{6}$, the CEF ground states are well separated from the first excited ones and identified as non-Kramers, i.e., carrying an orbital degeneracy larger than the minimum reachable under an electrostatic influence. Their respective degeneracies are $4\left(\Gamma_{8}\right), 3\left(\Gamma_{5}\right)$, and $4\left(\Gamma_{8}\right)$. However, in all three cases, the low-temperature specific-heat measurements yield values of the paramagnetic entropy much lower than expected from the degeneracy of these CEF ground states [12-15]. In the case of $\mathrm{CeB}_{6}$, a value consistent with the $\Gamma_{8}$ quadruplet is reached for temperatures one order of magnitude higher than the ordering one. This is possibly related to a Raman scattering observation: at low temperature, the cubic CEF quadruplet ground state spreads over an energy range of about $30 \mathrm{~K}^{\text {in }} \mathrm{CeB}_{6}[10,16]$. Similar entropy anomalies are observed as well in rare-earth-filled skutterudites [17-19]. These recurrent inconsistencies force to reconsider the effect of the CEF in the cage context. The group theory approach relies on a system with ideal point symmetry, whereas physical systems are necessarily imperfect in this regard, due, at least, to thermal excitations and zeropoint fluctuations. This issue should be most severe in cage compounds. Indeed, how relevant is an approach based on the 


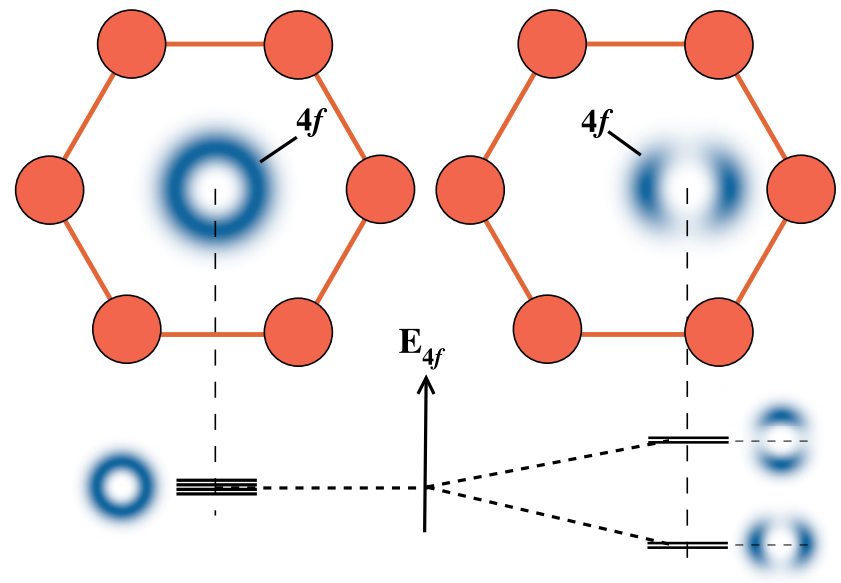

FIG. 1. Schematic illustrating the effect, inside a high-symmetry cage, of an off-center position for a rare-earth ion $\left(R^{3+}\right)$ with a non-Kramers CEF ground level. Outside the cage center, the nonKramers level is split (energy scale $E_{4 f}$ ). At low temperature, the offcenter position and its altered $4 f$ electrons distribution (schematized densities) are statistically favored.

point symmetries at the cage center if the magnetic atom can substantially deviate from it? Moreover, in the here considered systems, the point symmetry at the cage center is high. At the center, the CEF ground state is thus likely to display an excess of orbital degeneracy. Such orbital degeneracies are known to cause Jahn-Teller instabilities: at low temperature, the system tends to spontaneously reduce its symmetry, simultaneously lifting the orbital degeneracy and reducing the electrostatic energy. Many instances of Jahn-Teller effect are found in systems where $3 d$ ions occupy sites of octahedral symmetry, such as in perovskite and spinel structures, in which they cause a distortion of the octahedra and, collectively, of the crystal [20,21]. This cooperative kind of the Jahn-Teller effect is also found in rare-earth compounds [22], notably in high-symmetry insulators. These orbitally driven structural transitions are described considering a balance between the $4 f$ electrostatic energy and the elastic energy of the lattice. In crystals where high-symmetry cages accommodate loosely bound magnetic ions, the symmetry lowering does not require a distortion of the cage and, even less, of the crystal: it can be simply achieved with an offset magnetic ion (see Fig. 1). Accordingly, one can expect a Jahn-Teller effect to develop more easily in rare-earth cage systems than in conventional crystallographic structures. The work presented here attempts at answering these questions related to the specific $\mathrm{CEF}$ situation of cubic cage systems. The analysis is based on consideration of the lowest-order correction to the CEF cubic Hamiltonian for an offset ion. This results in a width for non-Kramers energy levels and in a specific, temperaturedependent, $4 f$ electronic term in the cage potential well.

\section{MODEL FOR AN ENCAGED $4 f$ ION}

\section{A. Definition of a cage system}

As a preliminary, an objective definition of the concept of rare-earth cage compound is required. In these systems, the crystal structure is supposed to leave some latitude for the displacement of the guest ion inside the cage. One should then expect large-amplitude vibrational modes (the so-called rattling), weakly coupled with the rest of the crystal. In order to identify a system as relevant to a cage-compound approach, beyond an analysis based on comparisons involving ionic radii and interatomic distances, one can define tangible experimental criteria: the Debye-Waller factor, the phonon dispersion curves, which are below examined.

\section{Debye-Waller factor}

As regards the displacement latitude, one direct information comes from the isotropic mean-square displacement of the rare earth $U_{\text {iso }}$, involved in the Debye-Waller factor. This information can be derived from neutron or x-ray diffraction. Some data can be found in the literature for the $\mathrm{RB}_{6}[23,24]$ and filled skutterudites from the antimonide series: $\mathrm{RFe}_{4} \mathrm{Sb}_{12}$ [25] and $\mathrm{ROs}_{4} \mathrm{Sb}_{12}$ [26,27]. At room temperature, $U_{\text {iso }}$ is about $0.005 \AA^{2}$ in light rare-earth hexaborides and between 0.02 and $0.04 \AA^{2}$ in the filled skutterudites, which yields a room-temperature root-mean-square (rms) amplitude of the displacement $\sigma$ of about $0.07 \AA$ in the $\mathrm{RB}_{6}$ and ranging between 0.1 and $0.2 \AA$ for the filled skutterudites. In both series, the values $U_{\text {iso }}$ for the rare-earth guest are typically one order of magnitude larger than the mean-square displacements for atoms from the cage. This gives a quantitative credit to the rattling picture in these series.

\section{Phonon dispersion}

In case of strictly local modes of the guests inside their cages, a flat, low-energy branch should appear when investigating the phonon dispersion. Actually, a large moving and coupled mass, as that of the rare earth, cannot leave unaffected the cage and lattice. The consequences of its movement can be easily derived using a classical, harmonic model: a linear chain of springs and masses [28]. In the upper part of Fig. 2, a variant of linear chain model is detailed where, in addition to the springs linking the guest (mass $m$ ) to the cage (stiffness $k_{0}$ ), and the cage to its neighbors (stiffness $K_{0}$ ), a spring of stiffness $K_{1}$ links the two halves (masses $M / 2$ ) of the cage. This allows to account for the elasticity of the cages in the description of a dispersion curve. The detail of the derivation of the dispersion relations is reported in Appendix A. In the graphs of Fig. 2, this model is confronted with inelastic neutron scattering data showing the fourfold axis longitudinal modes of $\mathrm{LaB}_{6}$ [29]. Large values for the stiffness $K_{1}$, with respect to $K_{0}$, are required to reproduce the physiognomy of the experimental data, which is well described in the limit of infinitely rigid cages $\left(K_{1} \rightarrow \infty\right.$ fit in Fig. 2$)$. Note that the only adjusted parameters are the two frequencies $\omega_{0}=\sqrt{2 k_{0} / m}$ and $\Omega_{0}=\sqrt{4 K_{0} / m}$, the mass ratio $m / M$ being fixed from the atomic masses of $\mathrm{La}$ and $99 \%{ }^{11} \mathrm{~B}$ enriched boron. It is thus shown that, for describing the two lowest dispersion curves of $\mathrm{LaB}_{6}$, one can safely consider rigid cages. This should generalize to the hole series of the hexaborides as, for instance, the experimental data [30] for $\mathrm{CeB}_{6}$ are very similar to those for $\mathrm{LaB}_{6}$. These dispersion curves can be interpreted as the anticrossing between the low-energy, flat 
Springs and masses model
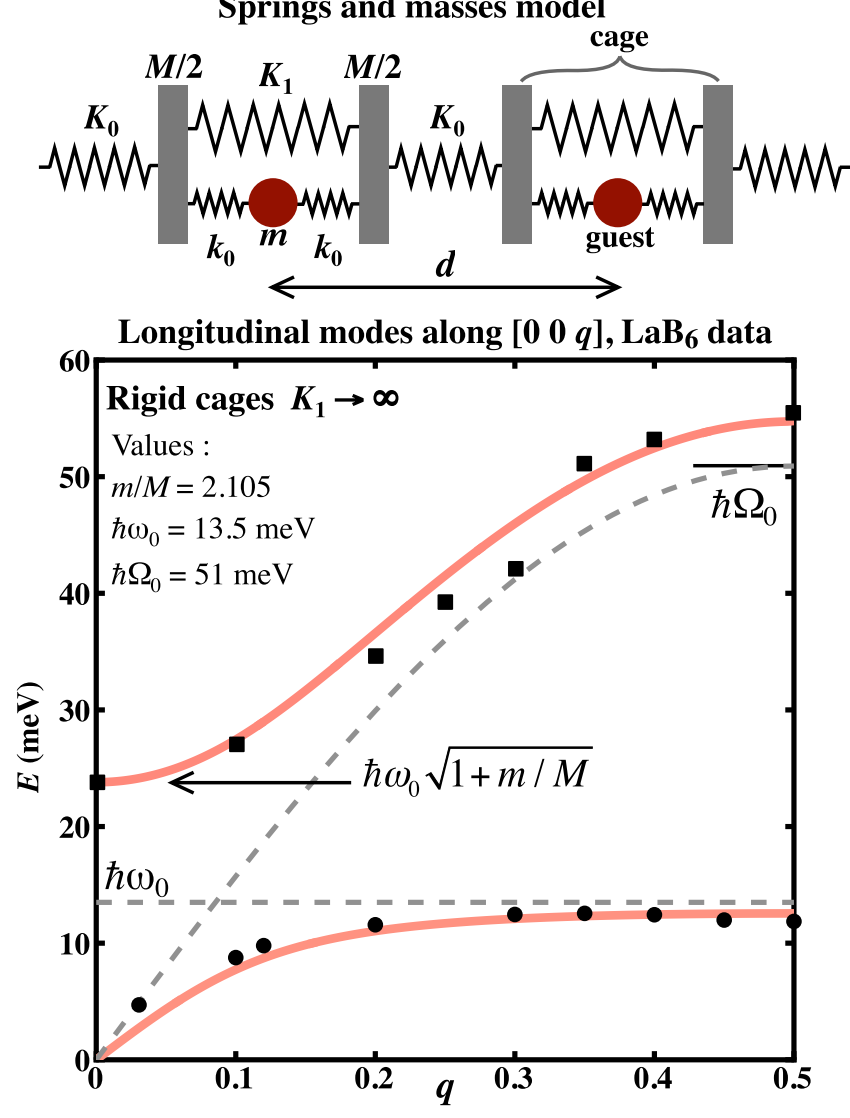

FIG. 2. Upper part, a classical chain of springs (stiffnesses $k_{0}$, $K_{0}$ ) and masses ( $m$ for the guest and $M$ for the cage) for describing longitudinal modes in cage systems, including a cage stiffness $K_{1}$. Lower part, the inelastic neutron scattering data for $\mathrm{LaB}_{6}$, from Ref. [29], confronted with computed dispersions curves (full lines) in the limit of perfectly rigid cages (see Appendix A). The dashed lines represent the underlying flat local mode of the guest, at frequency $\omega_{0}$, and acoustic branch for empty cages with maximum frequency $\Omega_{0}$.

branch of the guest vibration at frequency $\omega_{0}$ and the acoustic branch for a stiff lattice of empty cages with top frequency $\Omega_{0}$ (dotted lines in Fig. 2). The gap that opens has a width directly related to the ratio $m / M$ between the guest and cage masses. For the light boron cages in the hexaborides, this ratio is large as is the gap [29], whereas for filled skutterudites, the much heavier cages result in a smaller separation [31]. The experimental data for filled skutterudites are scarce, by lack of large single crystals, but for those available (see curves in Ref. [31]), the lowest branches reproduce the pattern observed for the hexaborides: an anticrossing between a low-energy "rattling" branch and the acoustic branch for a lattice of rigid cages. Using the harmonic approximation for an individual oscillator, the amplitude of the guest movement within the vibrational ground state is directly related to the level separation $\Delta E_{V}=\hbar \omega_{0}$. Along a given axis, the low-temperature rms deviation of the guest reads as $\sigma=\hbar / \sqrt{2 m \Delta E_{V}}$. As shown in Fig. 2, a value for $\Delta E_{V}$ can be obtained by looking at the flattened end of the acoustic dispersion branch. In filled skutterudites, this energy ranges between 4 and $9 \mathrm{meV}$
[31-33], while it is found slightly above $10 \mathrm{meV}$ in rare-earth hexaborides $\left(\approx 13 \mathrm{meV}\right.$ in $\mathrm{LaB}_{6}$ and $\mathrm{CeB}_{6}[29,30], \approx 11 \mathrm{meV}$ in $\mathrm{PrB}_{6}$ [34]). For these energies and an average lanthanide mass, the rms amplitude $\sigma$ ranges between 0.04 and $0.07 \AA$. Unsurprisingly, the low-temperature values are smaller than the room-temperature estimates derived from the diffraction data. Apart from this temperature effect, the spectroscopic and Debye-Waller investigations point to the same order of magnitude for the displacement inside the cages of skutterudites and hexaborides, of about $\frac{1}{10} \AA$. This analysis shows that the rareearth hexaborides and filled skutterudites share specific traits that define them as magnetic cage materials: large excursions of the guest inside the cage, with an amplitude of about $\frac{1}{10} \AA$, materializing in low-frequency vibrations of the rare earth. a close crystallographic environment of the rare earth, the cage, that can be viewed as essentially rigid when dealing with low-energy phenomena.

\section{B. Cage crystalline electric field}

The above analysis allows to consider the guest as mobile inside a rigid cage, with an excursion magnitude of a about $\frac{1}{10} \AA$. This is smaller than a rare-earth radius, which is in excess of $1 \AA$, but of the same magnitude as a typical $4 f$ shell radius. In relative terms, the movement of the $4 f$ shell with respect to its cage environment is substantial: a priori, the CEF difference between a centered and offset position of the $4 f$ shell cannot be neglected.

\section{Off-center crystal field}

In conventional compounds, the CEF is formalized by considering the point symmetry at the average position of the rare earth, that here identifies with the cage center. The cage center has the symmetries of a cubic point group: $T_{h}$ in case of a filled skutterudite and $O_{h}$ for a rare-earth hexaboride. The expression for the center CEF Hamiltonian $\mathcal{H}_{\mathrm{CEF}_{0}}$, describing the action of the CEF on the rare-earth ground-state $J$ multiplet strictly reflects the point symmetry of the rare-earth site [35,36]. In these high symmetries, only fourth- and sixth-order electric multipoles develop on the $4 f$ shell, but no quadrupoles: a degeneracy, larger than the Kramers minimum, is preserved for some of the CEF levels (Fig. 1, left side). The rare-earth ion being mobile, out of the cage center the symmetry of its environment is drastically reduced (right side of Fig. 1). The CEF acquires a dynamic character that, in the here considered rigid cage environment, is entirely due to the rare-earth movement. Considering the five orders of magnitude difference between the electron and rare-earth masses, it is the case to use a Born-Oppenheimer approximation, wherein the electronic $4 f$ wave functions continuously adapt to the position of the slowly moving rareearth ion. Local CEF stationary $4 f$ states, associated with a corrected Hamiltonian, are then a good approximation. This is accounted for by writing a position-dependent, static, CEF Hamiltonian $\mathcal{H}_{\mathrm{CEF}}(\boldsymbol{r})$, where, in addition to the main central term $\mathcal{H}_{\mathrm{CEF}_{0}}$, a corrective off-center $\mathcal{H}_{\mathrm{CEF}_{d}}$ is introduced:

$$
\mathcal{H}_{\mathrm{CEF}}(\boldsymbol{r})=\mathcal{H}_{\mathrm{CEF}_{0}}+\mathcal{H}_{\mathrm{CEF}_{d}}(\boldsymbol{r}) \text {. }
$$


The continuous symmetry lowering, associated with the change in the position $\boldsymbol{r}$ of the rare earth, is reminiscent of the treatment of magnetoelastic phenomena in rare-earth cubic compounds [37]: here, instead of being modulated by a strain, the corrective terms in the CEF Hamiltonian have to reflect the excursion out of the cage center. In both cases, the main correction is the interference in the Hamiltonian of second-order multipolar terms. This implies the emergence $4 f$ electric quadrupoles and the splitting of the non-Kramers CEF levels. The corrective $\mathcal{H}_{\mathrm{CEF}_{d}}$ term has to develop with the distance $r$ to the cage center. Restricting to the lowest order, the correction is quadratic both in the coordinates of the ion and in those, relative, of the $4 f$ electrons. It represents an energy term that has to be invariant under the symmetries of the rare-earth site. This imposes to couple a particular quadrupolar component with a combination of the $\boldsymbol{r}$ coordinates that has identical transformation properties. Moreover, for each quadrupolar irreducible representation, an individual coupling constant has to be introduced. According to these principles, in the case of a cubic symmetry at the cage center, the crystal field correction has necessarily the form

$$
\begin{aligned}
\mathcal{H}_{\mathrm{CEF}_{d}}(\boldsymbol{r})= & -D^{\gamma}\left[\left(3 z^{2}-r^{2}\right) O_{2}^{0}+3\left(x^{2}-y^{2}\right) O_{2}^{2}\right] \\
& -D^{\varepsilon}\left[(x y) P_{x y}+(y z) P_{y z}+(z x) P_{z x}\right],
\end{aligned}
$$

where $x, y$, and $z$ are the components, along the cubic axes, of the displacement $\boldsymbol{r}$ of the rare-earth nucleus from the center of the cage. $\left\{O_{2}^{0}, O_{2}^{2}\right\}$ and $\left\{P_{x y}, P_{y z}, P_{z x}\right\}$ are the quadrupolar operators transforming, respectively, as the $\gamma\left(\Gamma_{3}\right)$ and $\varepsilon\left(\Gamma_{5}\right)$ cubic irreducible representations [38]. In the $J$ manifold of the $4 f$ ion, they are conveniently written in terms of Stevens equivalents [39]. $D^{\gamma}$ and $D^{\varepsilon}$ are constants that, within a representation, define the magnitude of the coupling of the $4 f$ quadrupoles with the environment. In case of a displacement along a fourfold axis, only $D^{\gamma}$ is active, whereas along a threefold axis, it is $D^{\varepsilon}$.

\section{Broadening of the non-Kramers levels}

Inside the cage, as formalized by Eq. (2), the CEF scheme is no longer a stable feature of the rare earth, but depends on its position. The usual CEF scheme picture, with infinitely sharp energy levels, has to be abandoned: the non-Kramers cubic levels are broadened inside the cage, with an energy distribution that depends on the spatial distribution of the rare earth. At low temperature, this distribution is characteristic of the cage oscillator ground state and, as the temperature is increased, thermally excited vibrations should further spread it. In systems with large displacement coupling constants $\left(D^{\gamma}, D^{\varepsilon}\right)$ and small CEF splitting at the cage center, this broadening of the CEF levels might be competitive with their separation. This could explain anomalies reported in the neutron spectroscopy investigation of some filled skutterudites, where CEF excitations are absent [40], even at low temperature, or vanish rapidly while increasing the temperature $[3,8,9]$. The thermal broadening of non-Kramers levels is certainly not exclusive to rare-earth cage compounds. However, in rare-earth systems with more common crystallographic structures, a substantial deviation from the high symmetry of the rare-earth site requires short wave distortions of its environment. This would involve high-energy acoustic or optical phonons that are influential at temperatures typically competitive with the CEF levels spacing, above $100 \mathrm{~K}$. At these temperatures, CEF effects are drastically reduced and there would be no point in considering the CEF scheme broadening. Reciprocally, at lower temperatures, the broadening is negligible and it is usually legitimate to consider the ideal symmetry of the rare-earth site.

\section{Effect of a non-Kramers ground state on the paramagnetic properties}

For temperatures lower than the maximum splitting of the ground state inside the cage, which defines a characteristic energy scale, the properties will reflect the uneven population of the local CEF states. As the temperature is reduced, the state with lowest energy of the split multiplet is favored and the average magnetic entropy accordingly reduced, with consequences on the specific heat and the magnetic response (susceptibility). In this temperature range, an analysis based on the central $\mathrm{CEF}$ scheme is inappropriate for describing the experimental value of the magnetic entropy, magnetic susceptibility, and other CEF determined properties.

\section{Jahn-Teller mechanism}

According to Eq. (2), in case of a non-Kramers ground state, a splitting of this CEF multiplet develops quadratically with the distance $r$ to the center. This means that a lowerenergy electrostatic configuration can be achieved by moving away from the center. To be effective, this energy reduction also requires temperatures that reduce the statistical weight of the configurations with higher electrostatic energy: the energy scale associated with the cage splitting of the CEF ground state is also at play. This is the mechanism illustrated in Fig. 1: as the temperature is decreased, a centrifugal kind of Jahn-Teller effect can be expected to develop in systems with a central non-Kramers CEF ground state.

\section{Cage potential}

It appears that the distribution of the rare earth inside the cage is of critical influence on the CEF related properties, particularly in case of a non-Kramers, center ground state. The phonon dispersion analysis of Sec. II A 2 shows that one can consider the rare earth as exclusively coupled with a rigid cage, via the springs $k_{0}$ in Fig. 2. This means that the interaction between the cage and its guest can be treated separately, ignoring the rest of the crystal. In quantum mechanics, such an interaction is described by introducing a time-independent cage potential for the guest. Solving the Schrödinger equation for this potential well yields the sought after rare-earth distribution.

\section{Nonmagnetic potential well}

The confinement of the rare earth in the cage is not of magnetic origin. It is here formalized by introducing a potential well $V_{0}(\boldsymbol{r})$, where $\boldsymbol{r}$ refers to the displacement of the rareearth nucleus from the cage center. The systems of interest are metals, where the cage framework is built from strongly bound atoms. Those, in contrast with the rare-earth ion, have small motion latitude, the cage being considered here as 
perfectly rigid (see discussion in Sec. II A 2). As the rare-earth ion moves out of the center, its outer electrons reach those of the cage atoms: strongly repulsive forces develop, here simplified by considering an infinite barrier at the limits of the cage. This is not less realistic than a harmonic approximation and is of great practical interest when solving numerically the Schrödinger equation (see Appendix B). The actual shape of the barrier shares, at minimum, the symmetry elements of the center point group and may be rather complex. As only a few, low-energy vibration levels will be considered, a faithful description of the barrier is unnecessary. Instead, various degrees of approximation can be used, the most tractable ones being the cube and the sphere. Inside the cage, the bottom of the potential well cannot be flat, due to the electrostatic interaction between the rare-earth ion and charges from the neighboring atoms, bonds, and conduction electrons. These competing contributions may result in a complex shape for the bottom potential. However, in the cage systems we consider, the maximum deviation of the guest from the center is about one tenth of a rare-earth ionic radius. This is not large with respect to the crystallographic distances and a lowest-order description in the distance $r$ from the origin might be sufficient. Considering the central cubic point symmetry, this lowest order is necessarily isotropic, consisting in a simple quadratic term. To complement the infinite barrier, inside the cage the nonmagnetic potential is described as $V_{0}(r)=\alpha r^{2}$, where $\alpha$ is a constant, a priori positive in a metal, accounting for the different electrostatic contributions. The $V_{0}(\boldsymbol{r})$ term should have negligible temperature dependence in the temperature range of interest, below $100 \mathrm{~K}$. In the following, it is considered as independent from the temperature.

\section{CEF contribution to the potential well}

Due to the degeneracy of a non-Kramers CEF ground state, the $4 f$ electronic distribution can adjust to changes in its electrostatic environment. Here, the change is the consequence of the movement of the ion inside the cage and will contribute to the cage potential well with a specific $4 f$ term. This is formalized by the Hamiltonian term of Eq. (2), that has to be translated into an extra mean-field potential term $V_{4 f}(\boldsymbol{r}, T)$. In the original Born-Oppenheimer approximation, this question is treated adiabatically, considering only the lowest electronic energy level as the potential term. In the cage context, this lowest energy is not well separated from the excited electronic levels: due to the degenerate center CEF ground state, the energy separation goes to zero at the origin, thus realizing a conical intersection. One has then to face the difficulties of a nonadiabatic approach. One simplification comes from the fact that the rare-earth ion and its cage are not an isolated molecule, but belong to a crystal, moreover a metallic one. Then, even if the movement of the rare earth is so slow that the dynamic mixing of the local CEF states can be neglected, one has to consider the perturbing effect of the environment. In particular, frequent collisions should occur with conduction electrons, inducing transitions between the local CEF states. As a result, the electrostatic forces exerted on the rare-earth ion and associated potential term will rapidly fluctuate. The fast electronic fluctuations will have no effect on the massive rare earth which will be sensitive to an average that represents the $V_{4 f}(\boldsymbol{r}, T)$ mean field. For an extremely slow rare-earth movement, the statistics of the local $4 f$ states will approach a Boltzmann distribution defined by the temperature of the crystal. At constant temperature $T$, the additional work required to move the rare-earth ion, because of its evolutive $4 f$ aspherical distribution, equals the variation of free energy associated with the $4 f$ shell. In this nonadiabatic approximation, at a given temperature $T$, the magnetic part of the potential identifies with the local $4 f$ free energy:

$$
V_{4 f}(\boldsymbol{r}, T)=-k_{\mathrm{B}} T \ln \frac{Z(\boldsymbol{r}, T)}{2 J+1},
$$

where $Z(r, T)$ is a local partition function for the $4 f$ electronic states at a point $\boldsymbol{r}$ and temperature $T$. The division by $2 J+1$ is required in order to have a zero $V_{4 f}(\boldsymbol{r}, T)$ at $r=0$. In contrast with $V_{0}$, the $V_{4 f}$ potential is clearly temperature dependent. Indeed, at temperatures high with respect to the splitting inside the cage, all local CEF states are equally populated and the $V_{4 f}$ potential term flattens inside the cage. Conversely, at low temperature, $V_{4 f}(\boldsymbol{r})$ will follow the energy dependence of the lowest CEF level, decreasing quadratically with respect to the components of $\boldsymbol{r}$.

\section{SPHERICAL PROOF OF CONCEPT}

In principle, the discussion in the previous section allows to define the total cage potential at a given temperature:

$$
V(\boldsymbol{r}, T)=V_{0}(\boldsymbol{r})+V_{4 f}(\boldsymbol{r}, T) .
$$

From there, describing the movement of the enclosed rareearth ion requires to solve the time-independent Schrödinger equation for the mass $m$ of the rare-earth ion inside the potential well described by $V(\boldsymbol{r}, T)$. To describe the properties of a specific rare-earth system, one still has to detail the $V_{0}(\boldsymbol{r})$ term, which requires to choose an approximant for the cage shape, defining the infinite barrier in $V_{0}(\boldsymbol{r})$, and select a value for the constant $\alpha$. As regards the magnetic part of the potential well, the knowledge of the cage center CEF scheme is required or, at least, for a low-temperature description, an identification of the CEF ground state. Then, one has to select values for the $D^{\gamma}$ and $D^{\varepsilon}$ displacement-quadrupole couplings constants, involved in Eq. (2). After that, there remains the technical difficulty of integrating the Schrödinger equation for the possibly complex potential well.

Here, our purpose is not to investigate a specific compound, but to identify the physical consequences of a positiondependent CEF in the cage. This can be achieved using a further simplified model, provided it retains these essential features: the magnetic ion is trapped inside a cage, with high point symmetry at the center; the electronic ground state at the cage center is degenerate; the degenerate electronic states are split as the ion moves out of the center. By reducing the physical system to a spherical cage enclosing a rareearth ion, the first condition is met. Moreover, as the inner electric field produced by a uniformly charged sphere cancels, the $4 f$ electronic ground state should retain the full $2 J+1$ multiplet degeneracy. In order to lift the orbital degeneracy out of the cage center, one has to consider an additional, spherical charge density inside the cage. In a metal, this charge would correspond to that of the conduction electrons. In order 


\section{Spherical model}

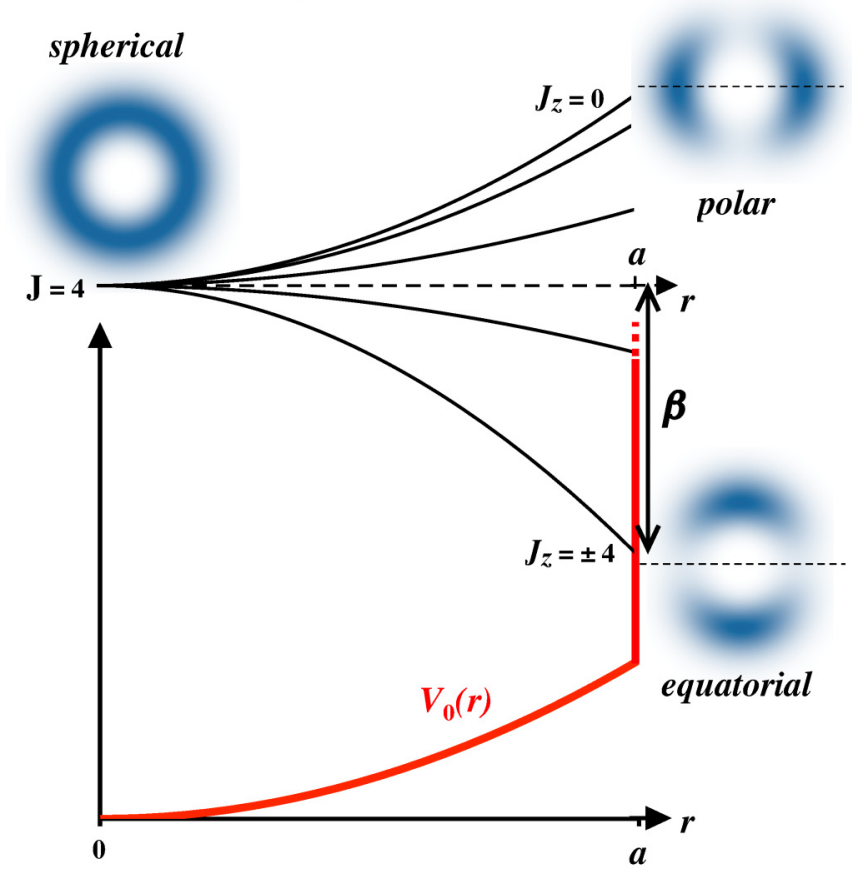

FIG. 3. Spherical model for a rare-earth ion with excursion latitude $a$ inside a rigid cage. The lower part shows the nonmagnetic potential $V_{0}(r)$ consisting in a quadratic bottom and infinite barrier at $r=a$. The upper part shows the splitting of the rare-earth multiplet (here exemplified by the $\operatorname{Pr}^{3+}$ ion, $J=4$ ), that yields a magnetic potential $V_{4 f}(r, T)$, at a distance $r$ from the center along the quantization $z$ axis. Here, the lowest energy is achieved for the doublet $J_{z}= \pm 4$ with "equatorial" distribution of the $4 f$ electrons and $\beta$ decrease in the electrostatic energy at $r=a$. The energy scales for the splitting and $V_{0}(r)$ are independent.

to introduce temperature-dependent effects, the cage and its guest also need to be coupled with a thermostat at $T$. This is also a role that can be deferred to the conduction electrons.

\section{A. Spherical potential well}

The spherical simplification reduces the nonmagnetic $V_{0}(\boldsymbol{r})$ potential to a form consisting in (see Fig. 3, lower part) an infinite spherical barrier at radius $a$, a restoring force, restricted to a quadratic term in the potential for $r<a$ : $V_{0}(\boldsymbol{r})=\alpha r^{2}$, where $\alpha$ is positive. The radius $a$ of the cage and the mass $m$ of the guest define the practical units for length $a$. Instead of using $\boldsymbol{r}$, the position in the cage is below referred to by $\rho=$ $\boldsymbol{r} / a$, in $a$ unit; energy $e . u .=\frac{\hbar^{2}}{2 m a^{2}}$, by reference to the energy levels of an infinite spherical well with radius $a$. The $V_{4 f}(\boldsymbol{r}, T)$ term reflects the electrostatic interactions between the $4 f$ shell and the conduction electrons. In the present spherical simplification, it should not reflect the quadrupolar anisotropy of Eq. (2), that arises in cubic symmetry for independent $D^{\gamma}$ and $D^{\varepsilon}$ coupling constants. Taking the quantization $z$ axis along the displacement direction, the isotropic reduction of the Hamiltonian term of Eq. (2) reads as

$$
\mathcal{H}_{\mathrm{CEF}_{d}}(\rho)=-\mathcal{D} \rho^{2} O_{2}^{0}=-\mathcal{D} \rho^{2}\left[3 J_{z}^{2}-J(J+1)\right],
$$

where $\mathcal{D}=2 D^{\gamma} a^{2}=24 D^{\varepsilon} a^{2}$.
This Hamiltonian describes the splitting of the $J$ multiplet outside the cage center (see Fig. 3, upper part). The eigenstates coincide with the $\left|J, J_{z}\right\rangle$ states, the ones with opposite $J_{z}$ projections being degenerate. Depending on the sign of $\mathcal{D}$, the local CEF ground state at $\rho$ will either correspond to the maximum projection doublet $J_{z}= \pm J$ or to the minimum, $J_{z}=0$ singlet, or $J_{z}= \pm \frac{1}{2}$ doublet. Following Stevens equivalent operators' method [39], the $4 f$ electrons' quadrupolar component along the displacement axis $Q_{z z}=\left\langle 3 z^{2}-r^{2}\right\rangle$ is directly related to the $J_{z}$ projection:

$$
Q_{z z}=\alpha_{J}\left\langle R_{4 f}^{2}\right\rangle\left\langle 3 J_{z}^{2}-J(J+1)\right\rangle
$$

where $\alpha_{J}$ is the Stevens second-order constant and $\left\langle R_{4 f}{ }^{2}\right\rangle$ the second moment for the $4 f$ radial wave function of the considered rare earth. From Eq. (5), it appears that the energy extrema correspond to an equatorial or polar distribution of the $4 f$ electrons along the displacement axis. To identify the configuration of lowest energy, more detail about the conduction electrons density is required. If a net excess of negative charge lies well inside the average $4 f$ radius, reduced to a central negative charge, a simple electrostatic calculation shows that an equatorial distribution of the $4 f$ electrons is favored. This means that the coupling constant $\mathcal{D}$ has to be negative for $\alpha_{J}$ positive (which occurs only for the heaviest tripositive rare earth) and, respectively positive, for $\alpha_{J}$ negative (which is the case for most tripositive rare-earth ions). In the following, it is assumed that the equatorial configuration is of the lowest energy, as represented on Fig. 3. For a given value of $\mathcal{D}, T$, and $\rho$, the local partition function $Z(\rho, T)$ can be computed, then the potential term, according to Eq. (3). Once the spherical potential $V(\rho, T)$ is defined, one can turn to solving the time-independent Schrödinger equation for the enclosed nucleus at a given temperature $T$. The angular parts of the eigenfunctions are the spherical harmonics $Y_{l}^{m}(\theta, \varphi)$, whereas the radial part $R_{n, l}(\rho)$ and energies $E_{n, l}$ require solving a differential equation specific to the considered potential. Except for very specific $l$ values and potential shapes, one is forced to resort to a numerical treatment (see Appendix B). In the following, the solutions to the Schrödinger equation are labeled using the usual atomic notations $n[l]$, the sorted sequence of the levels being $1 s, 2 p, 2 d$, etc. In all physically relevant cases, this energy sequence is maintained, with a $1 s$ vibration ground state.

\section{B. Thermodynamic and magnetic consequences}

\section{Energy scales}

In the hypothesis of a conduction electron's density favoring an equatorial distribution of the $4 f$ electrons around the displacement axis, the lowest energy corresponds to the $O_{2}^{0}$ value $Q_{0}=2 J^{2}-J$, for $\alpha_{J}$ negative (doublet, $J_{z}= \pm J$ ). For $\alpha_{J}$ positive, $Q_{0}=-J(J+1)$ for an integer $J$ (singlet, $J_{z}=0$ ), or $Q_{0}=3 / 4-J(J+1)$ for a half-integer $J$ (doublet, $\left.J_{z}= \pm 1 / 2\right)$. One can introduce the constant

$$
\beta=\mathcal{D} Q_{0}
$$

that equals the decrease in energy of the lowest $4 f$ level when the rare earth has maximum deviation from the center (see Fig. 3). As the temperature approaches the range of $\beta$, the local probabilities of occupation will start to segregate between the $4 f$ levels. This has necessarily an impact on the 
thermodynamic and magnetic properties which acquire a specific temperature dependence for $T$ lower than $\beta$. Estimating the order of magnitude of $\beta$ is crucial in order to decide wether it interferes with the energy scale of the vibrations, represented by the separation $\Delta E_{V}$ of the lowest vibration levels. To this regard, the only quantitative experimental indication comes from the example of $\mathrm{CeB}_{6} \cdot \mathrm{CeB}_{6}$ has a well-isolated $\mathrm{CEF} \Gamma_{8}$ quadruplet ground state, separated by $\Delta E_{\mathrm{CEF}}=46 \mathrm{meV}$ from the excited $\Gamma_{7}$ doublet [10]. Actually, $\Delta E_{\mathrm{CEF}}$ is temperature dependent, showing an increase of $1.24 \mathrm{meV}$ in the Raman CEF excitation [10] when cooling down from 300 to $4 \mathrm{~K}$. Ascribing this to the average lowering of the cage split $\Gamma_{8}$ level (the $\Gamma_{7}$ doublet cannot be split), the magnitude of the $\beta$ equivalent in the $\mathrm{CeB}_{6}$ case should be about $2 \mathrm{meV}$. As regards the energy scale of the vibration, for rare-earth hexaborides, experiments show that $\Delta E_{V}$ is slightly above $13 \mathrm{meV}$ for $\mathrm{LaB}_{6}$ (see Fig. 2). The $\mathrm{CeB}_{6}$ example is thus representative of this energy scale's hierarchy:

$$
\beta<\Delta E_{V}<\Delta E_{\mathrm{CEF}}
$$

This quantitative examination of $\mathrm{CeB}_{6}$ yields a $15 \%$ estimate for the ratio between $\beta$ and $\Delta E_{V}$. In this case, the effect of the splitting of the central ground-state multiplet will manifest itself at temperatures much lower than the $\Delta E_{V}$ vibration energy scale. This allows a further simplification, wherein the only considered cage distribution is that of the vibrational ground state. The below spherical illustration, with the praseodymium example, matches with the energy scales of $\mathrm{CeB}_{6}$ : it consists in a well-isolated, orbitally degenerate electronic ground state, with a $\beta$ cage splitting significantly smaller than the $\Delta E_{V}$ separation of the vibration levels. Beyond the $\mathrm{CeB}_{6}$ example, the other rare-earth hexaborides $\mathrm{PrB}_{6}$ and $\mathrm{NdB}_{6}$, for which the CEF scheme is well determined [11], also have a well-isolated non-Kramers CEF ground state. A hierarchy of energy scales, similar to the $\mathrm{CeB}_{6}$ case, is likely to apply. More contrasty is the situation of filled skutterudites. The anomalies, possibly related to the CEF, reported in their paramagnetic range, mostly occur at low temperature, typically below $30 \mathrm{~K}$. This is lower than the vibration energy scale $\Delta E_{V}$ that is inside the $50-100 \mathrm{~K}$ range, thus agreeing with the $\mathrm{CeB}_{6}$ example as regards the $\beta<\Delta E_{V}$ condition. However, as regards the amplitude of the CEF level spacing, all situations, with small or large $\Delta E_{\mathrm{CEF}}$ with respect to the other energy scales, can be found in these series. The analogy with the below spherical illustration has then to be judged on a case-by-case basis. As regards the calculation of macroscopic observables such as the internal energy, entropy, magnetic susceptibility, etc., note that the hypothesis of a local Boltzmann distribution (introduced in Sec. II C 2 for computing the magnetic contribution to the cage potential well) is not required. The measured system contains an extremely large number of equivalent cages which, considered as independent, realize a large ensemble for which a Boltzmann distribution is certainly applicable. As shown below in Sec. III C, in relative terms, the magnetic effect on the potential is small.

\section{Praseodymium example}

At a site of cubic symmetry, the CEF ground state of an ion with integer value of $J$ can be onefold, twofold, or threefold degenerate [36]. In case of a half-integer $J$, the ground state can be twofold or fourfold degenerate. The here relevant cubic $\mathrm{CEF}$ ground states are non-Kramers, i.e., carry an excess of orbital degeneracy. In case of an integer values of $J$, they are thus twofold or threefold degenerate and fourfold degenerate for a half-integer $J$. If, instead of a pseudospin, one considers a real rare-earth ion inside the spherical cage, the central $2 J+1$ degeneracy is necessarily higher than that of a cubic, non-Kramers CEF ground state. Nevertheless, for the sake of a quantitative illustration, we arbitrarily select the example of a $J=4$ multiplet that corresponds to the ground multiplet of $\mathrm{P} \mathrm{Pr}^{3+}$ ion (ninefold degenerate at the center). As the second-order Stevens coefficient $\alpha_{J}$ is negative for $\operatorname{Pr}^{3+}$, an equatorial distribution of the $4 f$ electrons corresponds to a positive $\left\langle O_{2}^{0}\right\rangle$. This means that the local lowest-energy level is the $J_{z}= \pm 4$ doublet and that the zero-temperature limit of $\left\langle O_{2}^{0}\right\rangle$ is $Q_{0}=28$ (see Fig. 3). Moreover, to get the minimum energy for this $4 f$ distribution, the $\mathcal{D}$ constant in Eq. (5) has to be positive. Then, one has to choose a value for $\mathcal{D}$. For the most simple, flat bottom, spherical well, the $1 s-2 p$ separation is 10.33 e.u., which means that a simple value $\beta=2$ e.u. agrees with the order of magnitude derived from the $\mathrm{CeB}_{6}$ example. In order to have $\beta=2$ e.u. for a $\operatorname{Pr}^{3+}$ guest, one has $\mathcal{D}=\beta / Q_{0}=1 / 14$ e.u.

\section{Calculation and results}

Another required parameter for the calculation is $\alpha$, that defines the quadratic bottom for the nonmagnetic potential $V_{0}(r)$. There is no experimental data that could help estimate the magnitude of $\alpha$. Two values, 0 for a flat bottom and 5 e.u. for a pronounced harmonic bottom, relatively to the $1 s-2 p$ separation, are used in the following calculations. Once the potential is defined at a given temperature $T$, solving the radial Schrödinger equation (see Appendix B) yields the radial wave function $R_{1,0}(\rho)$. The $4 f$ partition function at $\rho$ allows to define the local values for the internal energy $U_{4 f}(T, \rho)$, entropy $S_{4 f}(T, \rho)$, and specific heat $C_{V_{4 f}}(T, \rho)$. The averaged values $U_{4 f}(T), S_{4 f}\left(T\right.$, ), and $C_{V_{4 f}}(T, \rho)$, for the $1 s$ state are then obtained by numerical integration over the radial distribution. For instance, in the case of $U_{4 f}(T)$,

$$
U_{4 f}(T)=\int_{0}^{1} 4 \pi \rho^{2}\left|R_{1,0}(\rho)\right|^{2} U_{4 f}(T, \rho) d \rho .
$$

Figure 4 gives the temperature dependencies of these quantities, in the range defined by the $\beta$ value. The plot of the constant volume specific heat $C_{V}$ is obtained from a numerical derivative of $U_{4 f}$. The chosen value for $\beta$ has little impact on the potential well, then on the temperature variation of the $1 \mathrm{~s}$ vibration energy: in this temperature range, the variation of the total internal energy is essentially that of $U_{4 f}$.

In the upper part of Fig. 4, one can observe how the average $4 f$ energy is lowered for temperatures below $\beta / k_{\mathrm{B}}$. In a real system, with a non-Kramers CEF ground state at the center, this decrease in energy of the CEF ground state results in a larger transition energy to an unsplit excited CEF level. This is precisely what is observed via neutron and Raman scattering experiments in the example of $\mathrm{CeB}_{6}$ [10]. Note that the shift in energy in this spherical illustration represents only $27 \%$ of the $\beta$ value and about 5\% of the energy difference between the lowest $1 s$ and $2 p$ vibration energies. A better agreement 


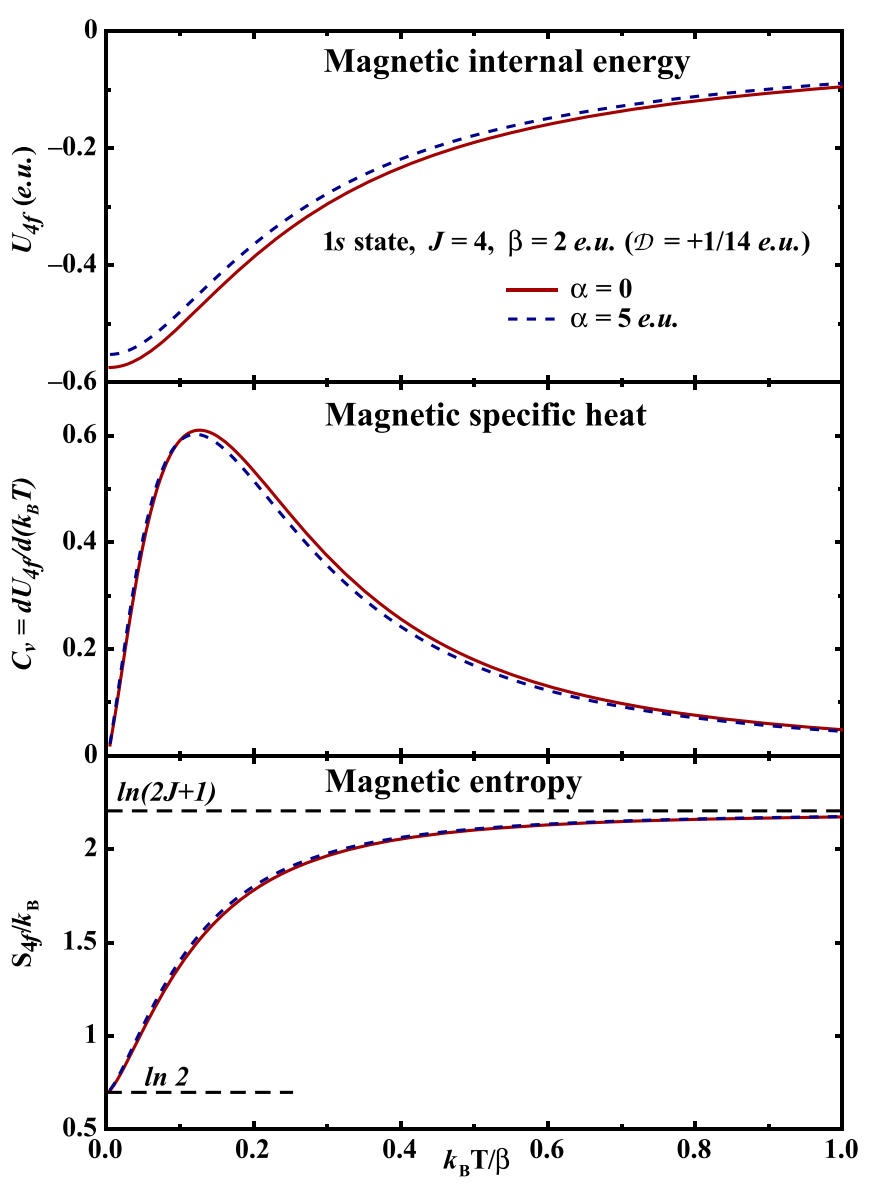

FIG. 4. Computed thermodynamic consequences, as function of the reduced temperature $k_{\mathrm{B}} T / \beta$, for a cage split $J=4$ multiplet in the spherical $1 s$ vibrational ground state. The full line curves are obtained for a nonmagnetic potential with flat bottom $(\alpha=0)$, whereas the dashed lines are for a quadratic bottom $(\alpha=5$ e.u.). $\beta$ is the amplitude of the energy decrease for the lowest CEF level at the cage limit. The upper part gives the temperature variation of the $4 f$ shell contribution to the internal energy. The middle and lower frames, respectively, show the associated specific heat and magnetic entropy.

with the order of magnitude derived from $\mathrm{CeB}_{6}$ example would require a larger $\beta$ value of about 6 e.u. As regards the specific heat, the $4 f$ energy lowering results in a Schottkytype anomaly that peaks at a temperature between 0.1 and $0.2 \beta / k_{\mathrm{B}}$. In systems where an ordering occurs at temperatures competitive with $\beta / k_{\mathrm{B}}$, only the higher-temperature part of the peak will appear on the specific-heat curves, the peak itself being concealed by the order. This is typically the case in rare-earth hexaborides where most elements order antiferromagnetically. Low-temperature Schottky-type anomalies are reported for rare-earth-filled skutterudites, notably for praseodymium compounds with nonmagnetic CEF ground state as $\mathrm{PrOs}_{4} \mathrm{Sb}_{12}$ [41] or $\mathrm{PrOs}_{4} \mathrm{P}_{12}$ [42]. These anomalies are interpreted as the effect of a low-lying CEF level above the $\Gamma_{1}$ singlet ground state. As illustrated here, an alternative would be to consider an isolated, cage split, nonmagnetic $\Gamma_{23}$ doublet ground state. Schottky anomalies are also observed to survive the ferromagnetic order in neodymium compounds such as $\mathrm{NdOs}_{4} \mathrm{Sb}_{12}$ [17], $\mathrm{NdOs}_{4} \mathrm{As}_{12}$ [19], or $\mathrm{NdRu}_{4} \mathrm{As}_{12}$ [43]. In all these examples, the crystal field ground state seems to be a non-Kramers quadruplet, therefore likely to be split in the cage context. The associated entropy (Fig. 4, lower part) shows that a temperature level of $\beta / k_{\mathrm{B}}$ is required to recover the entropy of the $J=4$ multiplet. A prolonged increase of the entropy in the paramagnetic range, with difficulties for achieving the well-defined degeneracy of a CEF ground state, is a frequent trait of rare-earth cage compounds. In the illustrating case of the $J=4$ multiplet, the choice of a positive value for $\mathcal{D}$ defines the doublet $J_{z}= \pm 4$ as the local CEF ground state at any point in the cage, except the center. This results in an average magnetic entropy that does not go to zero but to the finite value $k_{\mathrm{B}} \ln 2$ at zero temperature. Conversely, a negative $\mathcal{D}$ would have resulted in a singlet local CEF ground state and vanishing magnetic entropy at $0 \mathrm{~K}$. Another information brought by the calculation is the little influence of the bottom of the nonmagnetic cage potential, expressed via the values 0 (full line in Fig. 4) and 5 e.u. (dashed line) for $\alpha$. The positive $\alpha$ slightly reduces the extension of the $1 s$ wave functions, diminishing, at a given temperature, the CEF energy gain. However, the amplitude of the effect and overall aspect of the curves are very similar. The essential parameters of the model are the size of the cage $a$ and the CEF splitting amplitude $\beta$. Both define separate energy scales, $a$ acting on the guest vibration energy separation $\Delta E_{V}$ whereas $\beta$ determines the lower energy CEF effects.

\section{Magnetic susceptibility}

One most visible influence of the CEF scheme, when investigating rare-earth compounds, is the change in the magnetic susceptibility with respect to the degenerate $2 J+1$ groundstate multiplet. Similarly, an effect on the susceptibility can be expected in the $\beta / k_{\mathrm{B}}$ temperature range, where the population of the $4 f$ states evolves. To compute the magnetic susceptibility in the cage context, a Zeeman term needs to be added to the local CEF Hamiltonian of Eq. (1). In the spherical simplification, the CEF term at radius $\rho$ reduces to the uniaxial $O_{2}^{0}$ term [Eq. (5)] and has to be complemented with the Zeeman perturbation in order to write the $4 f$ Hamiltonian at $\rho$ :

$$
\mathcal{H}(\rho)=-\mathcal{D} \rho^{2} \frac{\hbar^{2}}{2 m a^{2}} O_{2}^{0}+\mu_{0} \mu_{\mathrm{B}} g_{J} \boldsymbol{H} \cdot \boldsymbol{J},
$$

where $g_{J}$ is the Landé factor and $\boldsymbol{H}$ the applied magnetic field. In the uniaxial symmetry, the local susceptibility tensor reduces to the susceptibilities parallel $\chi_{\|}$and perpendicular $\chi_{\perp}$ to the displacement axis. At given temperature $T$ and position $\rho, \chi_{\|}(T, \rho)$ and $\chi_{\perp}(T, \rho)$ can be computed from a perturbation approach or, as done here, via a linearization of the magnetization curve obtained from numerical diagonalization and Boltzmann statistics. Considering only the $1 s$ vibration state, the effective susceptibility results from a spherical average over all displacement directions. The averaged contribution from a radius $\rho$ reads as

$$
\chi(T, \rho)=\frac{\chi_{\|}(T, \rho)+2 \chi_{\perp}(T, \rho)}{3} .
$$

The total, isotropic, susceptibility $\chi(T)$ is then obtained by a second average of $\chi(T, \rho)$ over the $1 s$ radial distribution at temperature $T$ [see the example of Eq. (8)]. In practice, it is 


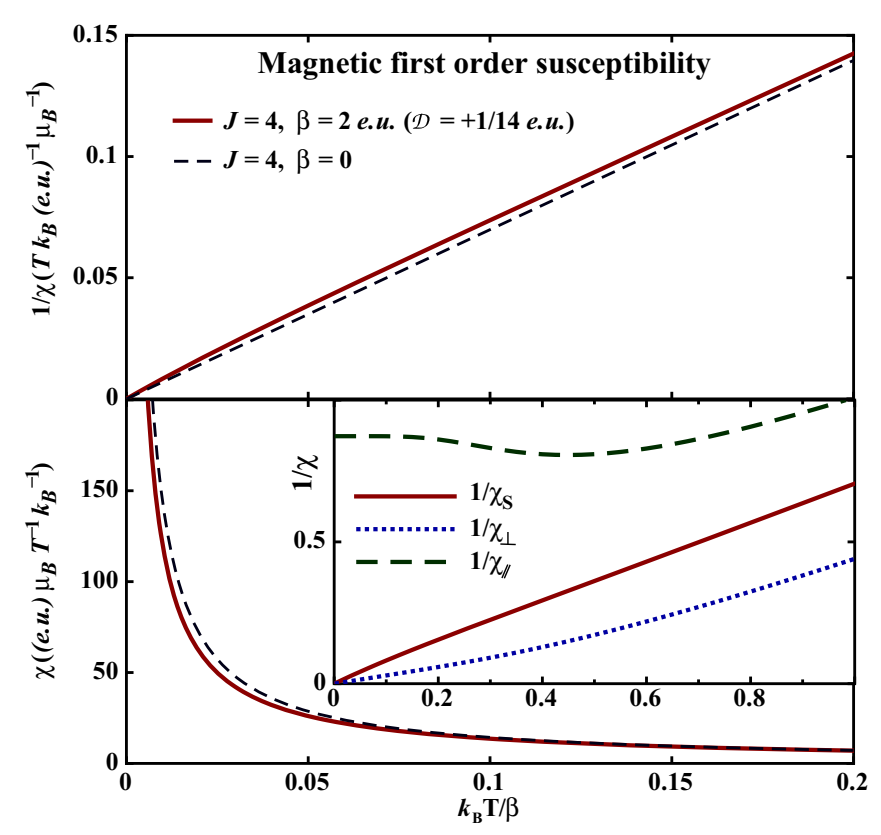

FIG. 5. Effect of the cage-split $J=4$ multiplet on the magnetic susceptibility $\chi$, as function of the reduced temperature $k_{\mathrm{B}} T / \beta$. The upper graph shows the inverse magnetic susceptibility, the lower one the direct susceptibility. The full line gives the result of the calculation for the $1 s$ state with $\mathcal{D}=\frac{1}{14} e . u$. and $\alpha=0$ (there is no visible difference for $\alpha=5$ e.u.), while the dashed line gives the Curie law for $J=4$. The inset shows the inverse susceptibilities, parallel $\chi_{\|}$and perpendicular $\chi_{\perp}$ to the displacement axis, as well as the resulting spherical average $\chi_{S}$, used as a standard curve (see text).

not necessary to compute $\chi(T, \rho)$ for each radius value. Since the scale of the local CEF scheme is entirely defined by $\mathcal{D} \rho^{2}$, a standard curve $\chi_{S}(T)$ can be computed once for all and then adapted to any particular value of $\rho$. In the present case, the calculation is done for $\mathcal{D}=\frac{1}{14} e . u$. and $\rho=1$ over and extended temperature range (inset of Fig. 5), thus defining $\chi_{S}(T)$. From this standard curve, $\chi(T, \rho)$ for $\rho<1$ is scaled as

$$
\chi(T, \rho)=\frac{\chi_{S}\left(T / \rho^{2}\right)}{\rho^{2}} .
$$

The results of Fig. 5 show a moderate effect of the cage splitting, with respect to a reference Curie law for $J=4$. The difference is visible only for temperatures much lower than $\beta / k_{\mathrm{B}}$. As usual for a CEF effect in a high-symmetry system, a reduction of the susceptibility is observed. Due to the hypothesis of a positive $\mathcal{D}$ value, the split multiplet has a local $J_{z}=$ \pm 4 , magnetic doublet ground state, that maintains a large susceptibility. A very different picture would arise for a negative $\mathcal{D}$ value, that selects a nonmagnetic local ground state. The effect of the quadratic bottom of the potential well is here imperceptible, at least in the considered cases $\alpha=0$ and 5 e.u..

\section{Centrifugal Jahn-Teller effect}

\section{Zero-temperature limit}

In order to identify the effects of the $V_{4 f}$ potential term, one can focus on the zero-temperature limit, where it has maximal amplitude and simplest analytical form. For $T$ going

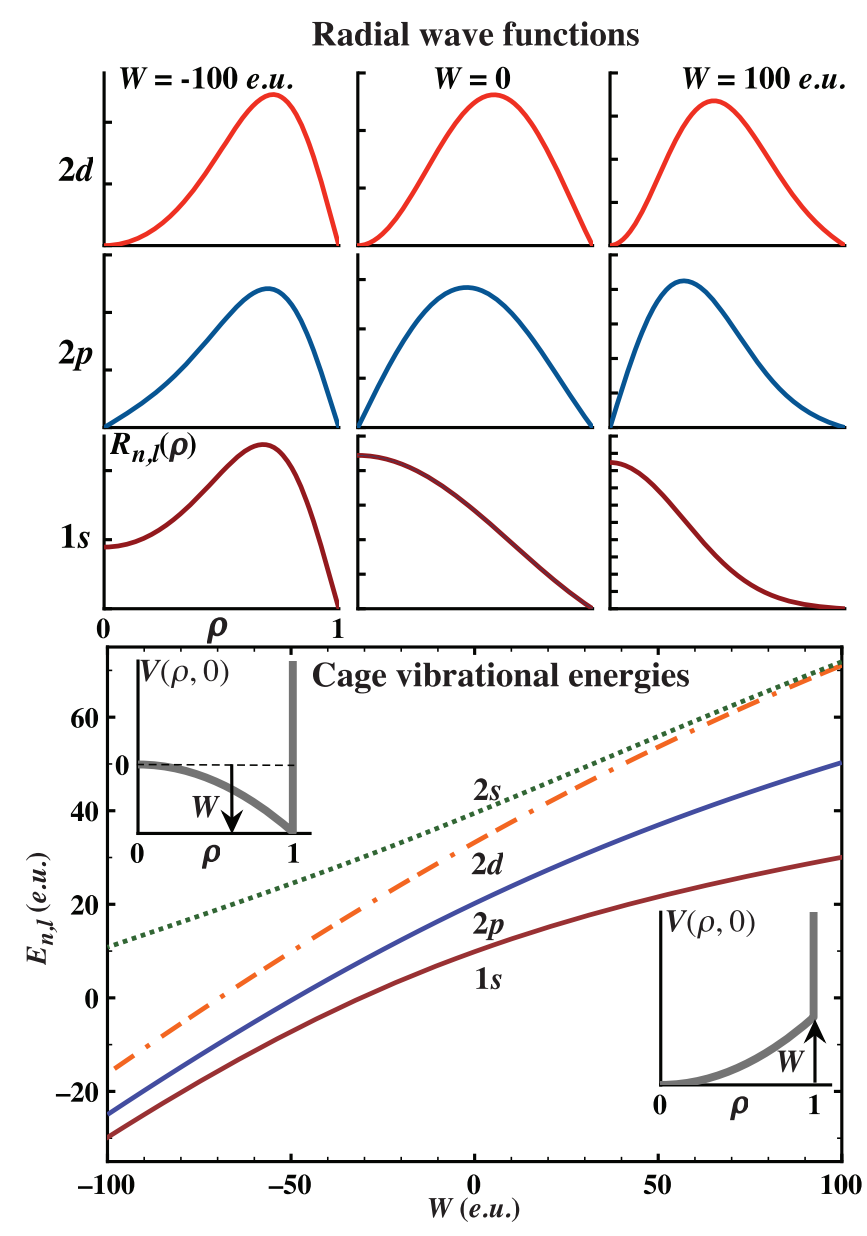

FIG. 6. Zero-temperature effect of the spherical well quadratic bottom, defined by the constant $W$. Lower part: energies of the four lowest oscillator levels $1 s, 2 p, 2 d$, and $2 s$, as functions of $W$ with the insets representing, as functions of the reduced radius $\rho$, wells for positive and negative $W$. The CEF contribution results in a $\beta$ shift of $W$ toward negative values. Upper part: sketches of the radial wave functions for the $1 s, 2 p$, and $2 d$ states, for a flat bottom $(W=0)$ and for opposite strong convexities $(W=100$ and -100 e.u. $)$.

to zero, in the set of quadrupolar values at $\rho, Q_{0}$ with its lowest energy is statistically dominant. In the zero-temperature limit, the magnetic potential simplifies to $V_{4 f}(\rho)=-\beta \rho^{2}$. Then, at $T=0$ and for $\rho<1$, the total cage potential writes as

$$
V(\rho, 0)=(\alpha-\beta) \rho^{2}=W \rho^{2} .
$$

Figure 6 shows the dependence on $W$ of the four lowestenergy levels: $1 s, 2 p, 2 d$, and $2 s$. For large positive $W$ values, the system tends to harmonicity, with a constant level separation and "accidental" $2 d-2 s$ degeneracy. The centrifugal $4 f$ term shifts $W$ the opposite way, toward negative values by the amount of $\beta$, which results in: a reduced energy difference between the three lowest levels, particularly between the singlet $1 s$ ground state and the first excited triplet $2 p$ states; radial wave functions that adapt to the centrifugal term. The $1 s$ ground state is the most affected (see the upper part of Fig. 6) as, from Eq. (B1), the centrifugal effect is exclusively of magnetic origin in case $l=0$. The concomitant reduction of energy separation and increase in the wave-function 
overlap increases the multipolar susceptibilities of the rareearth distribution. In particular, the dipolar and quadrupolar susceptibilities, respectively involving $1 s$ to $2 p$ and $1 s$ to $2 d$ matrix elements. This should facilitate low-temperature phenomena that involve a redistribution of the rare-earth ion inside the cage. For instance, the dipolar susceptibility is at play in the antiferromagnetic range of rare-earth hexaborides, where exchange-induced displacements of the rare earth (i.e., dipoles) determine the magnetic wave vector and the first-order kind of the magnetic transition $[44,45]$. The cage quadrupoles, analogously to the $4 f$ ones [37], should couple with the crystal strain modes and, via their associated susceptibilities, influence the elastic properties. This coupling with the rare-earth cage distribution also applies for a volume strain. Despite the above consideration of a perfectly rigid cage, in a real elastic system, an evolution in the guest radial distribution (see the sketches for the $1 s$ radial wave function in Fig. 6) will impact the cage and lattice volumes. The centrifugal term should also affect the lowest acoustic and optical phonon branches (see Fig. 2): the reduced $1 s-2 p$ spacing at $T=0$ means that, for all phonons involving the vibration of the rare-earth inside its cage, some softening will develop as the system is cooled.

\section{Thermal dependence}

To identify the above effects, the amplitude of the centrifugal term has been deliberately exaggerated: the horizontal scales of Fig. 6 go well beyond the orders of magnitude considered in Sec. III B. Here, we go back to the same $J=4$, $\beta=2$ e.u. and $\alpha=0$ or $\alpha=5$ e.u. values as in Sec. III B. Inspired by the $\mathrm{CeB}_{6}$ orders of magnitude, these values ensure more realistic predictions as regards the centrifugal effect and its temperature dependence. The calculation relies on the more delicate, nonadiabatic, approximation that allows to individually define a potential for the considered cage [Eq. (3)]. Figure 7 shows, as a direct illustration of the centrifugal effect, the temperature dependence of the second-order radial moment $\left\langle\rho^{2}\right\rangle$ for the $1 s$ state. The radial distribution expands as the temperature goes below $\beta / k_{\mathrm{B}}$. For $\beta=2$ e.u., the amplitude of the relative change in $\left\langle\rho^{2}\right\rangle$ reaches about $1.5 \%$. This amplitude is similar for the two considered nonmagnetic bottoms, defined by $\alpha=0$ and 5 e.u.. The absolute values of $\left\langle\rho^{2}\right\rangle$ for $\alpha=5$ e.u. are logically smaller because of the associated restoring force that opposes the Jahn-Teller centrifugal effect. This seems too small to be investigated by diffraction techniques through a Debye-Waller analysis, but high-precision dilatometry might be an option. Indeed, as the cage and crystal cannot be infinitely rigid, an isotropic change in the rare-earth distribution has necessarily an impact on the crystal volume. In an elastic cubic lattice of cages, since a volume strain $\varepsilon^{\alpha}=\varepsilon_{x x}+\varepsilon_{y y}+\varepsilon_{z z}$ and the secondorder radial moment of the guest $\left\langle\rho^{2}\right\rangle=\left\langle x^{2}\right\rangle+\left\langle y^{2}\right\rangle+\left\langle z^{2}\right\rangle$ transform identically, they can be phenomenologically related [46]. At the lowest-order description, a small change in $\left\langle\rho^{2}\right\rangle$ is linearly related to a volume strain: $\varepsilon^{\alpha}=\kappa\left(\left\langle\rho^{2}\right\rangle-\left\langle\rho_{0}^{2}\right\rangle\right)$, where $\left\langle\rho_{0}^{2}\right\rangle$ is the second-order radial moment in absence of the centrifugal effect. At this point, one cannot guess the magnitude and sign of the constant $\kappa$. Nevertheless, for a sufficiently large $\kappa$, a specific volume change and associated

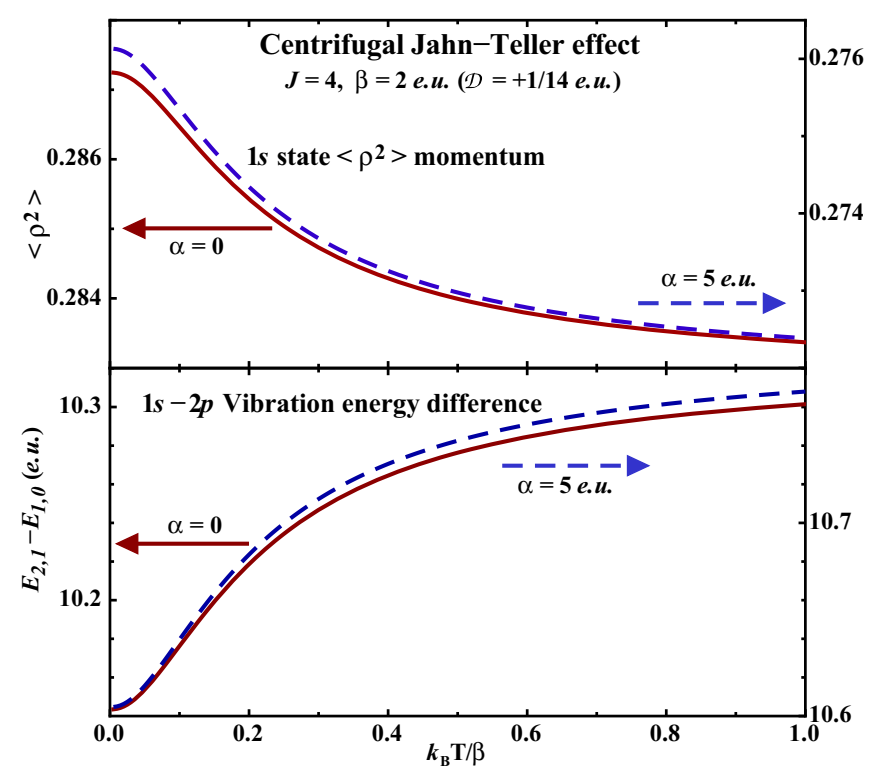

FIG. 7. Illustrations of the centrifugal Jahn-Teller effect in the spherical simplification within the $1 s$ state, for $J=4, \beta=2 e . u$. and two values, 0 (full lines) and 5 e.u. (dashed lines), of $\alpha$. The upper part materializes the centrifugal effect via the temperature dependence of the second-order radial moment $\left\langle\rho^{2}\right\rangle$ for the cage guest. The lower part shows the softening effect on the energy difference between the lowest vibration levels $1 s$ and $2 p$. The left vertical scales are for the flat bottom case, $\alpha=0$, whereas the right ones are for $\alpha=5$ e.u.. Left and right scales do not differ in amplitude, but are adapted by a shift.

thermal expansion anomaly should be detected in a cage system with a non-Kramers CEF ground state. There is scarce experimental data as regards the paramagnetic thermal expansion of filled skutterudites, but an x-ray investigation of rare-earth hexaborides shows noticeable differences between the nonmagnetic $\mathrm{LaB}_{6}$ and other elements in the series [47]. In particular, a peak develops in the linear thermal expansion coefficient of $\mathrm{CeB}_{6}, \mathrm{PrB}_{6}$, and $\mathrm{NdB}_{6}$ below $T=30 \mathrm{~K}$. It is to be noted that all three compounds have a non-Kramers CEF ground state $[10,11]$, which is the condition of realization of the centrifugal Jahn-Teller effect. Also, this anomaly occurs in a temperature range where, due to the large CEF level spacing, usual CEF volume effects should be absent. The lower part of Fig. 7 shows another effect of the centrifugal term in the cage potential, that is the decrease of the energy differences between the lowest-lying vibrational states. The graph displays the most relevant difference between the $1 s$ ground state and $2 p$ first excited level. This difference defines the vibration frequency of the "rattler" that, at low temperature, can be identified with $\omega_{0}$ in the harmonic approximation of Sec. II A 2. Here, for $\beta=2$ e.u., a softening of about $1.5 \%$ is predicted, that should directly reflect on the low-temperature dispersion curves (see Fig. 2), particularly on the flattened part of the "acoustic" branch and, at $q=0$, on the "optical" branch. Resolving a $1.5 \%$ softening is difficult using inelastic neutron scattering and infrared spectroscopy might be better adapted for detecting the small energy shift in the optical branch at $q=0$. The softening of acoustic phonons, close to 
the border zone, has been observed in $\mathrm{PrOs}_{4} \mathrm{Sb}_{12}$ [48] and in other filled skutterudites [33]. However, this softening is difficult to relate to the cage Jahn-Teller mechanism: the large magnitude of the effect, and its wide temperature range, do not fit with the energy scales in the above calculation.

\section{SUMMARY}

Inside a high-symmetry cage, an offset position of a rareearth ion splits its orbitally degenerate electronic levels. These levels thus acquire a dynamical width that reflects the distribution of the guest inside the cage. In case of an orbitally degenerate CEF ground state at the cage center, the associated energy width defines a temperature range, in the paramagnetic state, where very specific phenomena take place. Within this range, as the temperature is lowered, the lowest level of the split multiplet becomes statistically dominant. This allows a reduction of the $4 f$ energy. As this energy gain is obtained for an offset position, a centrifugal Jahn-Teller force is simultaneously exerted on the guest. In order to investigate the physical consequences of a cage-split electronic ground state, a simplified, spherical model has been used, where a $J=4$ multiplet enacts the degenerate level at the cage center. The experimental data on $\mathrm{CeB}_{6}$ offer a quantitative frame for this description, fixing a value for the energy width of the multiplet, that is much smaller than the separation between the lowest vibration levels. In this context, the only cage distribution that has to be considered is that of the singlet vibrational ground state. Thanks to this spherical simplification, the following is shown: for thermal energies below the electronic ground-state width, a Schottky-type anomaly develops in the specific heat, that evidences the decrease in the $4 f$ energy and entropy; the magnetic susceptibility is reduced with respect to the degenerate case; some volume anomaly should occur, reflecting the expansion of the rareearth distribution inside the cage; the centrifugal term reduces the rattling frequency of the guest, with resulting softening of low-energy phonons. This model also shows that the above conclusions are not dependent on the precise shape of the cage potential. Indeed, the two dominant energy scales in the problem are the separation $\Delta E_{V}$ between the lowest-lying and first excited vibration levels, which essentially depend on the size of the cage, and the smaller energy width of the electronic ground state $(\approx 2 \beta)$, that defines the amplitude and temperature range of the phenomena resulting from the split CEF ground state. This is encouraging as regards the description, in the paramagnetic range, of the macroscopic properties of a real cage system. Once defined the central CEF ground state, the rare-earth distribution in the lowest vibrational state is required for computing the specific heat, entropy, magnetic susceptibility, etc. In this purpose, the detail of the cage shape and associated potential well can be ignored. Indeed, the wave function for the singlet vibration ground state will be very similar for all highly symmetrical shapes of the potential well: harmonic well, spherical or cubic box, etc. What actually matters is the width of the rare-earth distribution and the magnitude of the quadrupole-displacement coupling constants. In this way, one may explain, by a pure crystal field mechanism, paramagnetic anomalies otherwise ascribed to exchange couplings, such as magnetic correlations or Kondo lattice related effects. An interesting perspective, at the price of an increased difficulty, is the description of paramagnetic properties under higher magnetic fields. The contrast in the local magnetic susceptibilities inside the cage should result in an anisotropic potential well: cage quadrupoles should emerge with an associated magnetostriction and influence on the magnetic anisotropy. Another, completely unexplored, aspect is the influence of a distributed crystal field on the ordering properties of a rare-earth compound. It is hoped that this work will encourage investigations in these directions.

\section{ACKNOWLEDGMENT}

The author would like to thank Dr. R.-M. Galéra and Dr. J. Robert for their critical reading of the manuscript.

\section{APPENDIX A: DISPERSION RELATIONS FOR A LINEAR CHAIN OF ELASTIC CAGES}

The chain, of period $d$, is defined in Fig. 2, through the sketch of the involved masses and springs. One starts by writing the classical equations of motion for the guest (mass $m$ ) and the two halves (masses $M / 2$ ) of the cage, subjected to the springs' restoring forces. Then, imposing the form of the displacements as imaginary exponentials describing propagating waves with angular frequency $\omega$ and wave vector $k=\frac{2 \pi}{d} q$, one gets the secular equation

$$
\left[\Omega_{0}^{2}[1-\cos (2 \pi q)]+2 \omega^{2} \frac{(1+\delta) \omega_{0}^{2}-\omega^{2}}{\omega^{2}-\omega_{0}{ }^{2}}\right]\left\{\Omega_{0}{ }^{2}[1+\cos (2 \pi q)]+2\left(\Omega_{1}{ }^{2}+\delta \omega_{0}^{2}-\omega^{2}\right)\right\}=\Omega_{0}{ }^{4} \sin (2 \pi q)^{2},
$$

where $\delta=\frac{m}{M}, \omega_{0}^{2}=\frac{2 k_{0}}{m}, \Omega_{0}^{2}=\frac{4 K_{0}}{M}, \Omega_{1}^{2}=\frac{4 K_{1}}{M}$.

Further parametrizing, introducing the reduced variable $x=\omega / \omega_{0}$, frequencies $\rho_{0}=\Omega_{0} / \omega_{0}$ and $\rho_{1}=\Omega_{1} / \omega_{0}$, replacing $\sin (2 \pi q)^{2}$ with $1-\cos (2 \pi q)^{2}=[1-\cos (2 \pi q)][1+\cos (2 \pi q)]$, some simplifications yield

$$
\rho_{0}^{2}[1-\cos (2 \pi q)]\left(\rho_{1}^{2}+\delta-x^{2}\right)\left(x^{2}-1\right)+x^{2}\left(\delta-x^{2}+1\right)\left\{\rho_{0}^{2}[1+\cos (2 \pi q)]+2\left(\delta+\rho_{1}^{2}-x^{2}\right)\right\}=0 .
$$

Isolating $\cos (2 \pi q)$, the general dispersion relation can be expressed as

$$
\cos (2 \pi q)=1-2 \frac{x^{2}}{\rho_{0}^{2}} \frac{\left(\delta-x^{2}+1\right)\left(\delta+\rho_{1}^{2}-x^{2}\right)}{\delta+\rho_{1}^{2}\left(1-x^{2}\right)}+\frac{2 x^{2}\left(x^{2}-\delta-1\right)}{\delta+\rho_{1}^{2}\left(1-x^{2}\right)}
$$


which, in the general case, yields three dispersion branches. In the limit of an infinitely soft cage $\left(\rho_{1}=0\right)$, the dispersion relation reads as

$$
\begin{aligned}
\cos (2 \pi q)= & 1-2 \frac{x^{2}}{\delta \rho_{0}^{2}}\left(\delta-x^{2}+1\right)\left(\delta-x^{2}\right) \\
& +\frac{2 x^{2}}{\delta}\left(x^{2}-\delta-1\right),
\end{aligned}
$$

whereas, for an infinitely rigid cage $\left(\rho_{1} \rightarrow+\infty\right)$, the dispersion relation simplifies to

$$
\cos (2 \pi q)=1-\frac{2}{\rho_{0}^{2}}\left(1+\frac{\delta}{1-x^{2}}\right) x^{2} .
$$

The cage becoming a single, rigid object, there are only two branches. This allows the fit of Fig. 2, according to the equation

$$
q=\frac{1}{2 \pi} \arccos \left[1-2\left(1+\frac{m}{M} \frac{\omega_{0}^{2}}{\omega_{0}^{2}-\omega^{2}}\right) \frac{\omega^{2}}{\Omega_{0}^{2}}\right] .
$$

\section{APPENDIX B: SOLVING THE RADIAL SCHRÖDINGER EQUATION}

The spherical symmetry allows to use the methods, easily found in quantum mechanics textbooks, developed for the quantum description of atoms. The angular part of the wave functions are the spherical harmonics $Y_{l}^{m}(\theta, \varphi)$, whereas the radial part $R_{n, l}(\rho)$ and energies $E_{n, l}$ require solving a differential equation specific to the considered potential, here
$V(\rho, T)$. It is convenient to introduce the radial function $u_{n, l}$ :

$$
u_{n, l}(\rho)=\rho R_{n, l}(\rho) .
$$

For $\rho<1$, in the case of the above-defined potential well, the sought after $u_{n, l}(\rho)$ functions have to satisfy the differential equation

$$
\frac{d^{2}}{d \rho^{2}} u_{n, l}=\left(\frac{l(l+1)}{\rho^{2}}+V(\rho, T)-E_{n, l}\right) u_{n, l},
$$

where $l$ is the orbital quantum number and $E_{n, l}$ the energy associated with $u_{n, l}$ (all energies are in $e . u$. units, as defined in Sec. III A). At the cage limit $\rho=1$, the infinite barrier imposes $u_{n, l}(1)=0$. At the origin, $u_{n, l}(\rho)$ has also to cancel as, otherwise, one would get a diverging radial wave function $R_{n, l}$ for $\rho$ going to zero. In the general case, the only practical option is a numerical treatment of Eq. (B1). For a given value of $l$ and numerically evaluating $V(\rho, T)$, one can iteratively refine a numerical solution, which yields the radial wave function $R_{n, l}$ and corresponding energy $E_{n, l}$. The calculations presented in this paper are realized using a simple Numerovtype method, the 0 to 1 radial interval being divided into 2000 equal segments. The convergence is tested by checking the stability of the $E_{n, l}$ eigenvalue, which is required to vary less than $10^{-8}$ e.u. between successive iterations. Obviously, the obtained $R_{n, l}$ need to be normalized so that $4 \pi \rho^{2}\left|R_{n, l}(\rho)\right|^{2}$ can be interpreted as the radial probability of presence of the nucleus.
[1] W. Jeitschko and D. Braun, Acta Crystallogr., Sect. B 33, 3401 (1977).

[2] E. D. Bauer, N. A. Frederick, P.-C. Ho, V. S. Zapf, and M. B. Maple, Phys. Rev. B 65, 100506(R) (2002).

[3] K. Iwasa, L. Hao, K. Kuwahara, M. Kohgi, S. R. Saha, H. Sugawara, Y. Aoki, H. Sato, T. Tayama, and T. Sakakibara, Phys. Rev. B 72, 024414 (2005).

[4] L. Keller, P. Fischer, T. Herrmannsdörfer, A. Dönni, H. Sugawara, T. Matsuda, K. Abe, Y. Aoki, and H. Sato, J. Alloys Compd. 323-324, 516 (2001), Proceedings of the 4th International Conference on $f$-Elements.

[5] J. Effantin, J. Rossat-Mignod, P. Burlet, H. Bartholin, S. Kunii, and T. Kasuya, J. Magn. Magn. Mater. 47-48, 145 (1985).

[6] M. Amara and R.-M. Galéra, Phys. Rev. Lett. 108, 026402 (2012).

[7] H. Bethe, Ann. Phys. 3, 133 (1929).

[8] J.-G. Park, D. T. Adroja, K. A. McEwen, M. Kohgi, and K. Iwasa, Phys. Rev. B 77, 085102 (2008).

[9] K. Iwasa, K. Saito, Y. Murakami, and H. Sugawara, Phys. Rev. B 79, 235113 (2009).

[10] E. Zirngiebl, B. Hillebrands, S. Blumenröder, G. Güntherodt, M. Loewenhaupt, J. M. Carpenter, K. Winzer, and Z. Fisk, Phys. Rev. B 30, 4052 (1984).

[11] M. Loewenhaupt and M. Prager, Z. Phys. B: Condens. Matter 62, 195 (1986).

[12] T. Fujita, M. Suzuki, T. Komatsubara, S. Kunii, T. Kasuya, and T. Ohtsuka, Solid State Commun. 35, 569 (1980).
[13] Y. Peysson, C. Ayache, J. Rossat Mignod, S. Kunii, and T. Kasuya, J. Phys. 47, 113 (1986).

[14] K. N. Lee, R. Bachmann, T. H. Geballe, and J. P. Maita, Phys. Rev. B 2, 4580 (1970).

[15] E. Westrum Jr., H. Clever, J. Andrews, and G. Feick, in Proceedings of the Fourth Conference on Rare Earth Research, Phoenix, Arizona, April 1964 (Gordon and Breach, New York, 1965), p. 597.

[16] M. Loewenhaupt, J. Carpenter, and C.-K. Loong, J. Magn. Magn. Mater. 52, 245 (1985).

[17] P.-C. Ho, W. M. Yuhasz, N. P. Butch, N. A. Frederick, T. A. Sayles, J. R. Jeffries, M. B. Maple, J. B. Betts, A. H. Lacerda, P. Rogl, and G. Giester, Phys. Rev. B 72, 094410 (2005).

[18] W. M. Yuhasz, N. P. Butch, T. A. Sayles, P.-C. Ho, J. R. Jeffries, T. Yanagisawa, N. A. Frederick, M. B. Maple, Z. Henkie, A. Pietraszko, S. K. McCall, M. W. McElfresh, and M. J. Fluss, Phys. Rev. B 73, 144409 (2006).

[19] T. Cichorek, A. Rudenko, P. Wisniewski, R. Wawryk, L. Kepinski, A. Pietraszko, and Z. Henkie, Phys. Rev. B 90, 195123 (2014).

[20] J. H. van Vleck, J. Chem. Phys. 7, 72 (1939).

[21] U. Öpik and M. H. L. Pryce, Proc. R. Soc. London, Ser. A 238, 425 (1957).

[22] G. Gehring and K. Gehring, Rep. Prog. Phys. 38, 1 (1975).

[23] D. Chernyshov, M. Smirnov, A. Menschikova, A. Mirgorodsky, and V. Trounov, Physica B (Amsterdam) 234, 146 (1997). 
Proceedings of the First European Conference on Neutron Scattering.

[24] Y. Takahashi, K. Ohshima, F. P. Okamura, S. Otani, and T. Tanaka, J. Phys. Soc. Jpn. 68, 2304 (1999).

[25] W. Schnelle, A. Leithe-Jasper, H. Rosner, R. Cardoso-Gil, R. Gumeniuk, D. Trots, J. A. Mydosh, and Y. Grin, Phys. Rev. B 77, 094421 (2008).

[26] K. Kaneko, N. Metoki, T. D. Matsuda, and M. Kohgi, J. Phys. Soc. Jpn. 75, 034701 (2006).

[27] J. Yamaura and Z. Hiroi, J. Phys. Soc. Jpn. 80, 054601 (2011).

[28] M. Christensen, A. B. Abrahamsen, N. B. Christensen, F. Juranyi, N. H. Andersen, K. Lefmann, J. Andreasson, C. R. H. Bahl, and B. B. Iversen, Nat. Mater. 7, 811 (2008).

[29] H. G. Smith, G. Dolling, S. Kunii, M. Kasaya, B. Liu, K. Takegahara, T. Kasuya, and T. Goto, Solid State Commun. 53, 15 (1985).

[30] S. Kunii, J. M. Effantin, and J. Rossat-Mingnod, J. Phys. Soc. Jpn. 66, 1029 (1997).

[31] C. H. Lee, I. Hase, H. Sugawara, H. Yoshizawa, and H. Sato, J. Phys. Soc. Jpn. 75, 123602 (2006).

[32] R. Viennois, L. Girard, D. Ravot, H. Mutka, M. Koza, F. Terki, S. Charar, and J. Tedenac, Physica B (Amsterdam) 350, E403 (2004), Proceedings of the Third European Conference on Neutron Scattering.

[33] K. Iwasa, Y. Mori, L. Hao, Y. Murakami, M. Kohgi, H. Sugawara, and H. Sato, J. Phys.: Conf. Ser. 92, 012122 (2007).

[34] M. Kohgi, K. Kuwahara, N. Ogita, M. Udagawa, and F. Iga, J. Phys. Soc. Jpn. 75, 085003 (2006).

[35] K. Takegahara, H. Harima, and A. Yanase, J. Phys. Soc. Jpn. 70, 1190 (2001).
[36] K. Lea, M. Leask, and W. Wolf, J. Phys. Chem. Solids 23, 1381 (1962).

[37] M. E. Mullen, B. Luthi, P. S. Wang, E. Bucher, L. D. Longinotti, J. P. Maita, and H. R. Ott, Phys. Rev. B 10, 186 (1974).

[38] P. Morin and D. Schmitt, in Quadrupolar Interactions and Magnetoelastic Effects in Rare Earth Intermetallic Compounds (Elsevier, Amsterdam, 1990), Chap. 1, pp. 1-132.

[39] K. Stevens, Proc. Phys. Soc. (London) A 65, 209 (1952).

[40] R. M. Galéra, C. Opagiste, M. Amara, M. Zbiri, and S. Rols, J. Phys.: Conf. Ser. 592, 012011 (2015).

[41] M. B. Maple, P.-C. Ho, V. S. Zapf, N. A. Frederick, E. D. Bauer, W. M. Yuhasz, F. M. Woodward, and J. W. Lynn, J. Phys. Soc. Jpn. 71, 23 (2002).

[42] K. Matsuhira, Y. Doi, M. Wakeshima, Y. Hinatsu, K. Kihou, C. Sekine, and I. Shirotani, Physica B (Amsterdam) 359-361, 977 (2005), Proceedings of the International Conference on Strongly Correlated Electron Systems.

[43] A. Rudenko, Z. Henkie, and T. Cichorek, Solid State Commun. 242, 21 (2016).

[44] M. Amara, S. E. Luca, R.-M. Galéra, F. Givord, C. Detlefs, and S. Kunii, Phys. Rev. B 72, 064447 (2005).

[45] M. Amara, R.-M. Galéra, I. Aviani, and F. Givord, Phys. Rev. B 82, 224411 (2010).

[46] E. R. Callen and H. B. Callen, Phys. Rev. 129, 578 (1963).

[47] V. Novikov, E. Pilipenko, and S. Bud'ko, Solid State Commun. 252, 51 (2017).

[48] K. Iwasa, M. Kohgi, H. Sugawara, and H. Sato, Physica B (Amsterdam) 378, 194 (2006), Proceedings of the International Conference on Strongly Correlated Electron Systems. 